\title{
Kelvin-Helmholtz Instability of Potential Vorticity Layers: A Route to Mixing
}

\author{
J. G. ESLER \\ Department of Mathematics, University College London, London, United Kingdom \\ L. M. Polvani \\ Department of Applied Physics and Applied Mathematics, and Department of Earth and Environmental Sciences, Columbia University, \\ New York, New York
}

(Manuscript received 6 June 2003, in final form 10 November 2003)

\section{ABSTRACT}

\begin{abstract}
The linear and nonlinear dynamics of layers of anomalously high potential vorticity (PV) are studied in detail. It is well known that PV layers are subject to slow, balanced, mixed barotropic-baroclinic instabilities. In this paper, it is shown that, in addition, PV layers are subject to a Kelvin-Helmholtz instability, operating on much smaller spatial and faster temporal scales.

For simplicity, spatially infinite layers of uniform anomalous PV are considered. Such layers are characterized by two key parameters: the ratio $\Delta q$ of their anomalous PV to the background PV, and the angle $\alpha$ between the layer and the direction of the ambient stratification gradient (in suitably scaled coordinates). It is found that Kelvin-Helmholtz appears, for certain values of $\alpha$, whenever $\Delta q>8$.

Of notable interest is the case of an initially vertical PV layer embedded in a weak ambient shear flow: for sufficiently large $\Delta q$, once the PV layer is tilted past a critical angle, Kelvin-Helmholtz instability becomes possible. It is argued that the breakdown of PV layers due to a Kelvin-Helmholtz instability induced by ambient shear might be an important systematic mechanism leading to irreversible mixing during stratosphere-troposphere exchange events. This is discussed in the context of an example of Kelvin-Helmholtz instability observed near a tropopause fold.
\end{abstract}

\section{Introduction}

It is now widely recognized that planetary and synoptic-scale Rossby waves in the atmosphere regularly "break" in a manner analogous to water waves on a beach, notably at the edge of the polar vortex (e.g., McIntyre and Palmer 1984) and at the tropopause (e.g., Appenzeller et al. 1996). On isentropic surfaces, individual wave breaking events are accompanied by the formation of thin filaments of potential vorticity (PV; Hoskins et al. 1985). The two-dimensional dynamics of these filaments has been studies in much detail (Dritschel 1989; Dritschel et al. 1991; Waugh and Dritschel 1991).

In reality, however, these filaments are three-dimensional layers of PV and, in general, they are tilted relative to the vertical direction. The above-mentioned two-dimensional studies have examined the behavior of such filaments in the special case in which they are exactly vertical. An alternative view of the dynamics of filaments, in which they are represented as an infinites-

Corresponding author address: J. G. Esler, Department of Mathematics, University College London, 25 Gower Street, London WC1E 6BT, United Kingdom.

E-mail: gavin@math.ucl.ac.uk imal perturbation to a horizontal tropopause, has also been proposed (Juckes 1995; Hakim et al. 2002). In this study, we generalize the problem and address the issue of stability for PV layers oriented at an arbitrary angle to the vertical direction.

The key result of this study is that, when the PV anomaly of the layer is sufficiently large, and when the angle to the vertical is within certain bounds, the PV layer becomes unstable to a Kelvin-Helmholtz (KH) instability, whose characteristics are quite different from those of the barotropic-baroclinic (BTBC) instabilities that have been considered in previous work. This finding suggests a potential mechanism for the mixing of a PV layer and its chemical constituents down to scales that are much smaller and in times that are much faster than balanced dynamics would allow.

We envisage this mechanism to occur naturally as PV layers are embedded in vertical shear flows. Although a PV layer may be nearly vertical in the early stages of its formation (Polvani and Saravanan 2000), it will become KH unstable as it is tilted over by the shear. Such tilting by external shears thus provides a spontaneous way for the flow to transition from slow, balanced dynamics to a fast, unbalanced evolution. In other words, the simple tilting by an external shear allows the flow 
to radically depart from the "slow manifold" (see, e.g., Ford et al. 2000, for a recent discussion).

The paper is organized as follows. In the next section we set up the problem, describe its geometry, define the key parameters, and then derive a version of the nonhydrostatic Boussinesq equations in an appropriate tilting coordinate system. This allows us to capture the dynamics of a tilting PV layer with a two-dimensional set of equations. In section 3 we construct the balanced flow associated with the tilting PV layer and then determine the regimes under which the layer is unstable to BTBC and/or KH modes in section 4. Numerical experiments are presented in section 5, illustrating the evolution of the PV layer under these instabilities. In the final section, the relevance of the $\mathrm{KH}$ instability to transport and mixing during stratosphere-troposphere exchange is discussed in the context of recent observations, discussed in Cho et al. (1999), following a tropopause fold.

\section{Derivation of the model equations}

To study the dynamics of an infinite tilted layer of anomalous PV embedded in a stratified, rotating environment with uniform buoyancy frequency $N$ and rotation rate $f / 2$, we start from the full three-dimensional Boussinesq equations for velocity $\mathbf{u}=(u, v, w)$, buoyancy $b$, and geopotential $\phi$ :

$$
\begin{aligned}
u_{t}+(\Lambda z+u) u_{x}+v u_{y}+w\left(u_{z}+\Lambda\right) & =-\phi_{x}+f v \\
v_{t}+(\Lambda z+u) v_{x}+v v_{y}+w v_{z} & =-\phi_{y}-f u \\
w_{t}+(\Lambda z+u) w_{x}+v w_{y}+w w_{z} & =-\phi_{z}+b \\
b_{t}+(\Lambda z+u) b_{x}+v b_{y}+w\left(b_{z}+N^{2}\right) & =f \Lambda v \\
u_{x}+v_{y}+w_{z} & =0
\end{aligned}
$$

Note that we have allowed for the presence of an ambient vertical shear $\overline{\mathbf{u}}=(\underline{\Lambda} z, 0,0)$, and that the stratified ambient buoyancy field $\bar{b}$ is in thermal wind balance with it; that is, we have $\bar{b}=N^{2} z-f \Lambda y$.

These equations are nondimensionalized using

$$
\begin{aligned}
& x, y \sim L, \quad z \sim H, \quad u, v \sim N H, \\
& w \sim N H^{2} / L, \quad t \sim L / N H, \quad \Lambda \sim N, \\
& b \sim N^{2} H, \quad \phi \sim N^{2} H^{2},
\end{aligned}
$$

where $H$ and $L$ are characteristic vertical and horizontal scales of the flow associated with the PV layer. In terms of the actual dimensions of the layer, $H=f L_{x} / N$, where $L_{x}$ is the width of the layer in the $x$ direction, and the horizontal scale $L$ will depend on the type of motion induced by the layer. Note that the layer width $L_{x}$ does not change as the layer is tilted by the shear flow. Notice also that we have avoided using an explicit velocity scale in (2), as the magnitude of the induced velocities will be set by the scale of the instabilities. We have also avoided using $f$ explicitly in the scalings as we do not wish to anticipate a priori the importance of rotation in the motions induced by the layer. These choices result in the nondimensional equations:

$$
\begin{aligned}
u_{t}+(\Lambda z+u) u_{x}+v u_{y}+w\left(u_{z}+\Lambda\right) & =-\phi_{x}+\epsilon v \\
v_{t}+(\Lambda z+u) v_{x}+v v_{y}+w v_{z} & =-\phi_{y}-\epsilon u \\
\delta^{2}\left[w_{t}+(\Lambda z+u) w_{x}+v w_{y}+w w_{z}\right] & =-\phi_{z}+b \\
b_{t}+(\Lambda z+u) b_{x}+v b_{y}+w\left(b_{z}+1\right) & =\epsilon \Lambda v, \quad \text { and } \\
u_{x}+v_{y}+w_{z} & =0 .
\end{aligned}
$$

Two nondimensional numbers appear in these equations:

$$
\epsilon=f L / N H=L / L_{x}
$$

is a measure of the relative importance of rotation and buoyancy effects ( $\epsilon^{-2}$ is the Burger number), and

$$
\delta=H / L
$$

is a typical ratio of the horizontal to vertical scales of the flow under consideration. The rationale behind our choice of scaling is that the PV layer may induce motion on different space and time scales depending on which instability is operative. Specifically, we envision two key scaling choices:

- a "slow" or "balanced" scaling, associated with BTBC instability, for which

$$
\delta=\frac{f}{N}, \quad \epsilon=1, \quad t \sim f^{-1}, \quad L \sim \frac{N H}{f}=L_{x} ;
$$

- a "fast" or "nonhydrostatic" scaling, associated with $\mathrm{KH}$ instability, for which

$$
\delta=1, \quad \epsilon=\frac{f}{N}, \quad t \sim N^{-1}, \quad L \sim H .
$$

Note that Prandtl's ratio is $\operatorname{Pr}=\delta \epsilon=f / N$ in either case and is thus always small.

The next step consists of taking advantage of the fact that the PV layer of interest is of infinite extent and simplifying the set of Eqs. (3) to reduce the dimensionality by one. To accomplish this, we introduce a suitably rotated coordinate system, whose geometry is illustrated in Fig. 1. At any instant, the PV layer is tilted at an angle $\alpha$ to the vertical in the correctly scaled coordinate system, in which the $z$ coordinate is "stretched" by the inverse of Prandtl's ratio $\operatorname{Pr}^{-1}=N / f$.

We define two new coordinates: $z$ perpendicular to the layer and $x$ parallel to the layer (see Fig. 1). Moving up or down the layer in the $x$ direction involves crossing stratification surfaces (surfaces of constant buoyancy), so the $x$ direction is dynamically distinct from the (horizontal) $y$ direction, which is parallel to both the layer and the stratification surfaces. Because stratification almost invariably acts to stabilize fluid flows, we postulate that the layer will invariably be subject to primary instabilities in the $y$ direction and that the evolution of these instabilities can thus be followed in the $y-z$ plane. 


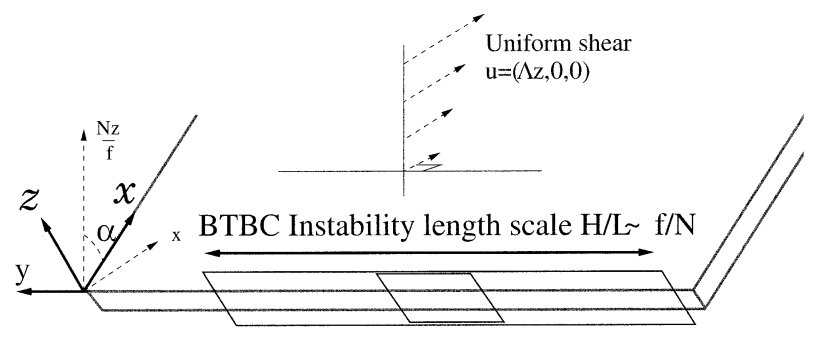

KH Instability length scale $\mathrm{H} / \mathrm{L} 1$

FIG. 1. Schematic illustration of a PV layer, showing the coordinate systems and the appropriate spatial scales for BTBC instability and $\mathrm{KH}$ instability.

In order to consider the evolution of the PV layer in two dimensions, we take $u, v, w, b$, and $\phi$ to be functions of $y, z$, and $t$ alone; that is,

$u=u(y, z, t), \quad v=v(y, z, t), \quad w=w(y, z, t)$,

$b=b(y, z, t), \quad \phi=\phi(y, z, t)$,

where $z$ is the rotating coordinate perpendicular to the PV layer (see Fig. 1) and is defined by

$$
z \equiv \Gamma z-\epsilon x \text {. }
$$

Here, $\Gamma \equiv \tan \alpha=\tan \alpha_{0}+\epsilon \Lambda t$, where $\alpha_{0}$ is the initial angle between the layer and the vertical in scaled coordinates. Under this transformation the Eqs. (3) become

$$
\begin{aligned}
u_{t}+v u_{y}+\mathcal{W} u_{z}+\Lambda w & =\epsilon \phi_{z}+\epsilon v, \\
v_{t}+v v_{y}+\mathcal{W} v_{z} & =-\phi_{y}-\epsilon u, \\
\delta^{2}\left(w_{t}+v w_{y}+\mathcal{W} w_{z}\right) & =-\Gamma \phi_{z}+b, \\
b_{t}+v b_{y}+\mathcal{W} b_{z}+w & =\epsilon \Lambda v, \quad \text { and } \\
v_{y}+\mathcal{W}_{z} & =0,
\end{aligned}
$$

where $\mathcal{U}$ and $\mathcal{W}$ are the velocities along and perpendicular to the tilting layer, respectively, and are given by

$$
\mathcal{U}=\Gamma u+\epsilon \delta^{2} w \text { and } \mathcal{W}=\Gamma w-\epsilon u .
$$

Combining (8) and (10), while recalling that $\Gamma=\Gamma(t)$, we get

$$
\begin{aligned}
\mathcal{U}_{t}+v \mathcal{U}_{y}+\mathcal{W} \mathcal{U}_{z}= & \epsilon \Gamma v+\epsilon b-\Lambda \mathcal{W}, \\
\delta^{2}\left(\mathcal{W}_{t}+v \mathcal{W}_{y}+\mathcal{W} \mathcal{W}_{z}\right)= & -\left(\operatorname{Pr}^{2}+\Gamma^{2}\right) \phi_{z} \\
& +\Gamma b-\epsilon^{2} v .
\end{aligned}
$$

For the purposes of numerical integration, it is helpful to recast (8)-(12) into three prognostic equations. This can be achieved by constructing a $y-z$ vorticity:

$$
\zeta \equiv \delta^{2} \mathcal{W}_{y}-\left(\operatorname{Pr}^{2}+\Gamma^{2}\right) v_{z}
$$

which satisfies the equation

$$
\zeta_{t}+v \zeta_{y}+\mathcal{W} \zeta_{z}=-2 \epsilon \Gamma \Lambda v_{z}+\Gamma b_{y}+\epsilon \Gamma \mathcal{U}_{z}
$$

The $v$ and $\mathcal{W}$ velocities can be obtained from the $y-z$ vorticity $\zeta$ by inverting

$$
\delta^{2} \psi_{y y}+\left(\operatorname{Pr}^{2}+\Gamma^{2}\right) \psi_{z z}=\zeta,
$$

where $\psi$ is the streamfunction for $v$ and $\mathcal{W}$ and satisfies

$$
\psi_{y}=\mathcal{W} \text { and } \psi_{z}=-v \text {. }
$$

The final set of equations, to be used for time stepping in the numerical model integrations, is then

$$
\begin{aligned}
\zeta_{t}+v \zeta_{y}+\mathcal{W} \zeta_{z} & =-2 \epsilon \Gamma \Lambda v_{z}+\Gamma b_{y}+\epsilon \Gamma \mathcal{U}_{z}, \\
\mathcal{u}_{t}+v \mathcal{U}_{y}+\mathcal{W} \mathcal{U}_{z} & =\epsilon \Gamma v+\epsilon b-\Lambda \mathcal{W}, \quad \text { and } \\
b_{t}+v b_{y}+\mathcal{W} b_{z} & =\epsilon \Lambda v-\frac{\Gamma \mathcal{W}+\epsilon \mathcal{U}}{\operatorname{Pr}^{2}+\Gamma^{2}}
\end{aligned}
$$

These equations are similar to the equations for twodimensional, nonhydrostatic Boussinesq flow in the $x$ $z$ plane (as described, e.g., by Esler et al. 1999) except for the addition of terms on the right-hand side that reflect the fact the coordinate system has been tilted.

\section{Balanced evolution of a tilting PV layer}

One particular solution of the model equations (20)(22) above represents the balanced flow and buoyancy field associated with a tilting, but otherwise unperturbed PV layer. In this section we show that, in the case where the layer is tilting due to (weak) uniform vertical shear, a balanced solution exists so that the flow and buoyancy field evolve "in balance" even as the layer tilts.

In the nonhydrostatic Boussinesq equations the PV is defined as

$$
q=(f \mathbf{k}+\boldsymbol{\nabla} \times \mathbf{u}) \cdot \boldsymbol{\nabla}(\bar{b}+b),
$$

and in the tilted coordinate system this becomes

$$
\begin{aligned}
q= & 1-\Lambda^{2}+\Gamma b_{z}+\frac{\zeta}{\operatorname{Pr}^{2}+\Gamma^{2}}-\Lambda \mathcal{U}_{z} \\
& +\frac{1}{\epsilon}\left(b_{y} \mathcal{U}_{z}-b_{z} \mathcal{U}_{y}-\frac{\Gamma \mathcal{U}_{y}}{\operatorname{Pr}^{2}+\Gamma^{2}}+\Lambda b_{y}\right) .
\end{aligned}
$$

We shall refer to the nondimensional units of $q$ as background PV units (BPVU) where 1 BPVU $\equiv f N^{2}$. In the absence of friction and diabatic effects, $q$ can be shown to satisfy the appropriate PV advection equation:

$$
\frac{\partial q}{\partial t}+v \frac{\partial q}{\partial y}+\mathcal{W} \frac{\partial q}{\partial z}=0 .
$$

The conservation equation ensures that if the PV is initially a function of $z$ alone, that is, $q(y, z, 0)=q(z)$, it retains the same distribution even as it is tilted by the shear, so that $q=q(z)=q\left[z\left(\Gamma_{0}+\Lambda t\right)-\epsilon x\right]$ for all time. In particular, this is the case for a uniform PV layer satisfying

$$
q= \begin{cases}q_{0} & |z|>\frac{h}{2} \\ \Delta q+q_{0} & |z|<\frac{h}{2} .\end{cases}
$$


Here $q_{0}=1-\Lambda^{2}$ is the PV due to the ambient stratification plus the vertical shear, and $\Delta q$ is the anomalous PV characterizing the layer.

Although the problem of inverting an arbitrary distribution of PV $q=q(y, z)$ is highly nontrivial and imprecisely defined, ${ }^{1}$ in the limit of weak of vertical shear $\Lambda \ll 1$ it is relatively straightforward to invert the simple distribution (26) to obtain a balanced velocity and buoyancy distribution. The balanced $v, b$, and $\mathcal{U}$ fields due to the layer can be found as follows. Exploiting the fact that $\mathcal{W}=\psi_{y} \equiv 0$, the $y$-independent version of Eqs. (20)-(22) can be written

$$
\begin{aligned}
v_{z t} & =-\frac{\epsilon \Gamma \mathcal{U}_{z}}{\operatorname{Pr}^{2}+\Gamma^{2}}, \\
b_{z t} & =\epsilon \Lambda v_{z}-\frac{\epsilon \mathcal{U}_{z}}{\operatorname{Pr}^{2}+\Gamma^{2}}, \\
\mathcal{U}_{z t} & =\epsilon\left(\Gamma v_{z}+b_{z}\right) .
\end{aligned}
$$

To derive an evolving balanced solution from these equations, it may be assumed that the rate at which the balanced fields must adjust to remain in balance is comparable to the rate at which the layer is tilting. Hence, a new time variable $T=\epsilon \Lambda t$ can be introduced with associated nondimensional units $\Lambda^{-1} f^{-1}$. Next, we expand each variable in powers of $\Lambda$ :

$$
\begin{aligned}
& v(Z, T)=v^{(0)}+\Lambda v^{(1)}+\Lambda^{2} v^{(2)}+\cdots \\
& b(Z, T)=b^{(0)}+\Lambda b^{(1)}+\Lambda^{2} b^{(2)}+\cdots \\
& \mathcal{U}(Z, T)=\mathcal{U}^{(0)}+\Lambda \mathcal{U}^{(1)}+\Lambda^{2} \mathcal{U}^{(2)}+\cdots .
\end{aligned}
$$

At leading order, the solution is easily shown to be

$$
\begin{aligned}
& b^{(0)}= \begin{cases}\Delta q \frac{\Gamma z}{1+\Gamma^{2}} & |z|<\frac{h}{2} \\
\Delta q \frac{\Gamma h}{2\left(1+\Gamma^{2}\right)} & z>\frac{h}{2} \\
-\Delta q \frac{\Gamma h}{2\left(1+\Gamma^{2}\right)} & z<-\frac{h}{2},\end{cases} \\
& v^{(0)}= \begin{cases}-\Delta q \frac{z}{1+\Gamma^{2}} & |z|<\frac{h}{2} \\
-\Delta q \frac{h}{2\left(1+\Gamma^{2}\right)} & z>\frac{h}{2} \\
\Delta q \frac{h}{2\left(1+\Gamma^{2}\right)} & z<-\frac{h}{2},\end{cases} \\
& \mathcal{U}^{(0)}=0 .
\end{aligned}
$$

This leading order solution is also the exact balanced

\footnotetext{
${ }^{1}$ Investing an arbitrary distribution $q(y, z)$ appears to be analogous to the much-discussed problem of inverting two-dimensional PV distributions in the shallow water equations (McIntyre and Norton 2000). If the distribution of PV is $y$ independent, however, a distribution of the form $q(x, z)$ can be inverted (in the limit of weak $\Lambda$ ) by solving a Monge-Ampere equation (e.g., Esler et al. 1999).
}

solution for a nontilting PV layer. As the layer tilts, and $\Gamma \equiv \tan \alpha_{0}+T$ increases, the layer's buoyancy and velocity distributions are adjusted due to advection in the $x$ direction by the velocity component $\mathcal{U}$. The latter can be shown to enter the balanced solution at the next order. Inside the layer $(|z|<h / 2)$,

$$
\begin{gathered}
b_{z}^{(1)}=0, \quad v_{z}^{(1)}=0, \\
\mathcal{U}_{z}^{(1)}=\frac{-2\left(\operatorname{Pr}^{2}+\Gamma^{2}\right)}{\left(\Gamma^{2}+1\right)^{2}} \Delta q,
\end{gathered}
$$

and $b_{z}^{(1)}=\boldsymbol{v}_{z}^{(1)}=\mathcal{U}_{z}^{(1)}=0$ outside the layer. It is necessary to obtain the solution at second order if $\mathcal{U}$ is to remain close to (32) as the layer tilts. At this order inside the layer we get

$$
\begin{aligned}
v_{z}^{(2)}= & \left(6 \Gamma^{4}-2 \Gamma^{2}-2 \operatorname{Pr}^{2}+10 \operatorname{Pr}^{2} \Gamma^{2}\right)\left(\Gamma^{2}+1\right)^{-4} \Delta q \\
b_{z}^{(2)}= & \left(-2 \Gamma^{4}+2 \Gamma^{2}+6 \operatorname{Pr}^{2}-2 \operatorname{Pr}^{2} \Gamma^{2}-4\right) \\
& \times\left(\Gamma^{2}+1\right)^{-4} \Gamma \Delta q \\
\mathcal{U}_{z}^{(2)}= & 0 .
\end{aligned}
$$

If Eqs. (27)-(29) are initialized with the balanced solutions up to second order, that is, $v=v^{(0)}+\Lambda^{2} v^{(2)}$, $b=b^{(0)}+\Lambda^{2} b^{(2)}, \mathcal{U}=\Lambda \mathcal{U}^{(1)}$, the velocity and buoyancy fields will remain within $O(\Lambda)$ of the leading order balance (31) even as the layer tilts an $O(1)$ amount. These are therefore suitable balanced buoyancy and velocity fields with which to initialize our model in section 5.

\section{Linear stability analysis}

\section{a. Condition for barotropic-baroclinic instability}

To determine the linear stability of a PV layer (26) with arbitrary $\Delta q$ and $\alpha$ would require a detailed numerical analysis of Eqs. (20) and (21). However, instructive analytic progress is easily made in the quasigeostrophic limit $(\delta, \Delta q \rightarrow 0)$. To further simplify matters, throughout this section we assume that the PV layer is nontilting $(\Lambda=0)$. As we will show, under suitable rescaling the problem turns out to be mathematically identical to that of the stability of a two-dimensional filament of vorticity as first studied by Rayleigh (1894). The dynamics of two-dimensional vorticity filaments have been widely studied in the geophysical literature (e.g., Dritschel 1989; Dritschel et al. 1991), as the same equations describe the dynamics of barotropic or vertical ( $\alpha=0$ ) PV layers of arbitrary $\Delta q$ (subject to $z$-independent disturbances). Notably, the effects of external shear, strain, and rotation on the filament stability and the consequent implications have been discussed. In the absence of these external flows, however, the two-dimensional filament/vertical PV layer is found to be unstable to disturbances with horizontal wavelength greater than $\approx 4.9 L$, where $L$ is the width of the layer, with the fastest growing disturbance having a wavelength around $7.9 L$ and a growth rate $\approx 0.2 \Delta q f$ (Drazin and Reid 1981, p. 146). 
Once the layer is tilted, its PV induces both velocity and buoyancy perturbations, and $\Delta q$ scales out of the problem only in the quasigeostrophic limit $\Delta q \rightarrow 0$. The quasigeostrophic PV equation is recovered from Eqs. (3) with $\Lambda=0$ by first assuming hydrostatic balance $(\delta=0)$. Exploiting the fact that $u, v, w$, and $b$ are at most $O(\Delta q)$, and that time can be rescaled as $\tilde{t}=\Delta q t$, to leading order each variable is expanded in powers of $\Delta q$. This yields geostrophic balance at leading order, and standard manipulation of the first-order terms results in

$$
\begin{aligned}
\left(\frac{\partial}{\partial \tilde{t}}-\phi_{y} \frac{\partial}{\partial x}+\phi_{x} \frac{\partial}{\partial y}\right) \tilde{q} & =0, \\
\tilde{q} & =\phi_{x x}+\phi_{y y}+\epsilon^{2} \phi_{z z} \\
& = \begin{cases}1, & \left|\Gamma_{z}-\epsilon x\right|<h / 2, \\
0, & \left|\Gamma_{z}-\epsilon x\right|>h / 2 .\end{cases}
\end{aligned}
$$

From the arguments of section 2, the primary instability of the layer will be independent of the $x$ coordinate. Transforming coordinates and assuming that the disturbance depends only on $y$ and $z$, we obtain

$$
\begin{aligned}
\left(\frac{\partial}{\partial \tilde{t}}+\epsilon \phi_{y} \frac{\partial}{\partial z}-\epsilon \phi_{z} \frac{\partial}{\partial y}\right) \tilde{q} & =0, \\
\tilde{q} & =\phi_{y y}+\epsilon^{2}\left(1+\Gamma^{2}\right) \phi_{z z} \\
& = \begin{cases}1, & |z|<h / 2, \\
0, & |z|>h / 2 .\end{cases}
\end{aligned}
$$

A further rescaling,

$$
\begin{aligned}
\hat{\phi} & =\epsilon^{2}\left(1+\Gamma^{2}\right) \phi, \quad \hat{y}=\epsilon\left(1+\Gamma^{2}\right)^{1 / 2} y, \\
\hat{t} & =\left(1+\Gamma^{2}\right)^{-1 / 2} \tilde{t},
\end{aligned}
$$

allows this equation to be written

$$
\begin{aligned}
\left(\frac{\partial}{\partial \hat{t}}-\hat{\phi}_{z} \frac{\partial}{\partial \hat{y}}+\hat{\phi}_{\hat{y}} \frac{\partial}{\partial z}\right) \tilde{q} & =0, \\
\tilde{q} & =\hat{\phi}_{\hat{y} \hat{y}}+\hat{\phi}_{z z} \\
& = \begin{cases}1, & |z|<h / 2, \\
0, & |z|>h / 2 .\end{cases}
\end{aligned}
$$

With Eq. (37) we have recovered the two-dimensional vorticity equation [Eq. (35) with $\Gamma=0$ ], with $-z$ and $\hat{y}$ in the roles of $x$ and $y$. The rescaling of time and distance reveals that as the layer is tilted, the time scale on which the instability develops increases with $\sec \alpha$ $\equiv\left(1+\Gamma^{2}\right)^{1 / 2}$, whereas the length scale of the unstable waves decreases in proportion to $\cos \alpha$. The BTBC instability of a PV layer therefore is gradually suppressed as the layer is tilted. This is perhaps not surprising since, in the limit $\alpha \rightarrow 90^{\circ}$, the velocity induced by the anomalous PV in the layer vanishes, and only the buoyancy fields carries a signature of the PV in the layer.

Of course, the above analysis applies only to the quasigeostrophic limit $\Delta q \rightarrow 0$. When $\Delta q$ is finite, however, it does not scale out of the problem, and away from the $\Delta q \rightarrow 0$ limit the normal mode structure depends on $\Delta q$. In the numerical calculations in section 5 below we will briefly investigate this dependence.

\section{b. Condition for Kelvin-Helmholtz instability}

The Miles-Howard condition for Kelvin-Helmholtz instability (Miles 1961; Howard 1961) states that a necessary condition for $\mathrm{KH}$ instability is that somewhere in the flow, the Richardson number

$$
\mathrm{Ri} \equiv \frac{\bar{b}_{z}+b_{z}}{u_{z}^{2}+v_{z}^{2}}<\frac{1}{4} .
$$

This condition applies exactly to our Eqs. (20)-(22) in the absence of rotation $(\epsilon=0)$. However, because $\mathrm{KH}$ instability has an aspect ratio of order unity $(\delta \sim 1)$, the effect of rotation on the developing waves is very weak $(\epsilon \sim \operatorname{Pr})$; that is, KH instability is described by the fast scaling given by (5) above. As Pr is typically very small in the atmosphere and oceans, we can use the Miles-Howard criterion to diagnose when the leading-order balanced response to a PV layer given by (31) becomes KH unstable.

For a layer of uniform PV, as given by (26), the Richardson number $\mathrm{Ri}_{\mathrm{PV}}$ within the layer is given, in terms of the parameters $\Delta q$ and $\Gamma \equiv \tan \alpha$ and to leading order in the vertical shear $\Lambda$, by the expression

$$
\mathrm{Ri}_{\mathrm{PV}}=\frac{1+\Gamma b_{z}}{\Gamma^{2} v_{z}^{2}}=\frac{\left(\Gamma^{2}+1\right)^{2}+\Delta q \Gamma^{2}\left(\Gamma^{2}+1\right)}{\Gamma^{2} \Delta q^{2}} .
$$

Figure 2 is a contour plot of $\mathrm{Ri}_{\mathrm{PV}}$ as a function of $\Delta q$ and $\alpha$. The Miles-Howard stability boundary $\mathrm{Ri}_{\mathrm{PV}}=1 /$ 4 is given by the thick solid contour, with locations above this line in the regime diagram subject to $\mathrm{KH}$ instability. The dashed line indicates the angle of tilt $\alpha_{m}$ corresponding to minimum $\mathrm{Ri}$ at a given value of $\Delta q$. It is easily shown that $\alpha_{m}$ satisfies

$$
\tan \alpha_{m}=\Gamma_{m}=(1+\Delta q)^{-1 / 4} \text {. }
$$

Note that $\alpha_{m}$ tends toward $45^{\circ}$ as $\Delta q \rightarrow 0$.

A simple expression for the minimum value of $\Delta q$ for which $\mathrm{KH}$ instability may occur can be found by solving

$$
\frac{\left(\Gamma_{m}^{2}+1\right)^{2}+\Delta q \Gamma_{m}^{2}\left(\Gamma_{m}^{2}+1\right)}{\Gamma_{m}^{2} \Delta q^{2}}=\frac{1}{4}
$$

whose relevant root yields

$$
\Delta q=8 .
$$

As is clear from Fig. 2, KH instability is therefore only possible for some angles $\alpha$ when $\Delta q>8$. For the mar- 


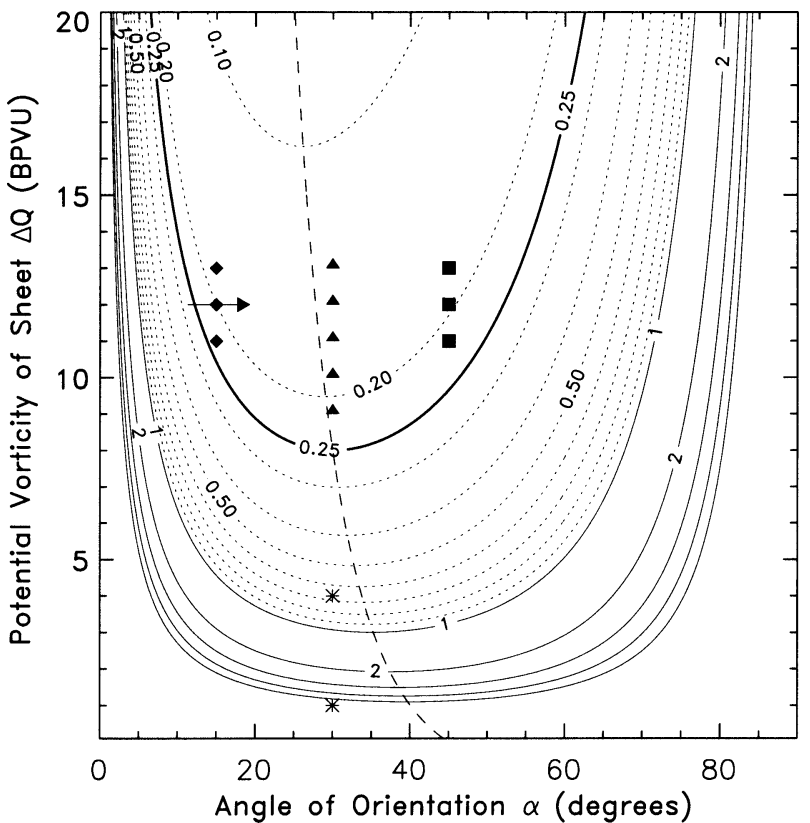

FIG. 2. The Richardson number Ri as a function of the angle of orientation $\alpha \equiv \tan ^{-1} \Gamma$ and anomalous PV in the layer $\Delta q$. The Miles-Howard stability boundary $\mathrm{Ri}=0.25$ is shown by the thick solid line. The dotted lines have contour interval $0.1(\mathrm{Ri}=0.1$ to $1.0)$ and the thin solid lines have contour interval 1 ( $\mathrm{Ri}=1$ to 5 ). The dashed line shows the angle of minimum Ri for a given $\Delta q$. The symbols show the locations of the numerical experiments described in section 5 (the arrow corresponding to the tilting Kelvin-Helmholtz experiment in Fig. 6).

ginally critical case $\Delta q=8$, it turns out that $\mathrm{Ri}_{\mathrm{PV}}=1 /$ 4 for an angle to the vertical $\alpha_{m}$ of $30^{\circ}$.

A similar analysis can be applied to the case of layers of anomalously low PV, with $-1<\Delta q<0$. Potential vorticity layers with $\Delta q$ in this range may occur when tropospheric air is transported into the stratosphere. From (39) it is easily shown that the minimum Richardson number for $\Delta q$ in this range is $\mathrm{Ri}_{\mathrm{PV}}=1$, occurring as $\Delta q \rightarrow-1$ and $\alpha \rightarrow 90^{\circ}$. Hence, uniform low $\mathrm{PV}$ layers are not subject to $\mathrm{KH}$ instability. Recall, in addition, that for $\Delta q<-1$, the $\mathrm{PV}$ in the layer will be opposite in sign to the background PV. Such layers, which might result from transport across the equator will, of course, be subject to inertial instability.

Traditionally, KH instability is discussed for flows with arbitrary vertical shear $U(z)$ and stratification $N(z)$. However, for balanced, rotating, stratified flows, $U$ and $N$ are determined by the PV distribution, which thus controls the Richardson number of the flow. It is such an example of PV-based KH instability that is investigated in the numerical experiments presented next.

\section{Numerical results and interpretation}

In this section we present results from numerical integrations of Eqs. (20)-(22) in a domain that is periodic in both the $y$ and $z$ directions, with dimensions
$2 \pi L^{*} \times 2 \pi H^{*}$. The geometry of the domain relative to the tilted layer is illustrated in Fig. 1. The model has been adapted from the pseudo-spectral code of Bartello (1995) and was used in a different configuration in Esler et al. (1999). The resolution employed in the experiments described below is $128 \times 256$ Fourier wavenumbers, in conjunction with a grid of $384 \times 768$ points. The greater resolution in the $Z$ direction is used to achieve a near-isotropic resolution within the Kelvin-Helmholtz billows in the experiments described below. A $\nabla^{4}$ hyperdiffusion is applied to the $\zeta, \mathcal{U}$, and $b$ fields to inhibit numerical instabilities. The time step employed is in the range 0.001-0.01 nondimensional time units, depending on the value of $\Delta q$ (note that the units of time are either $N^{-1}$ or $f^{-1}$, depending on the scaling choices described in section 2).

The numerical experiments are initialized with initial conditions based on the balanced state described in section $3, v=v^{(0)}+\Lambda^{2} v^{(2)}, b=b^{(0)}+\Lambda^{2} b^{(2)}, \mathcal{U}=\Lambda \mathcal{U}^{(1)}$ plus some small noise to allow instabilities to develop. Note that $\Lambda=0$ in many of the experiments described below. To satisfy the constraints of periodicity in the $z$ direction, it is necessary to modify the initial PV distribution slightly, as the domain-integrated PV anomaly must be zero to allow periodicity in $v$ and $b$. The total PV outside the layer is therefore not $q_{0}$ as in the idealized case discussed in section 4 but $q_{0}-\Delta q h /\left(H^{*}-\right.$ $h)$. Several numerical tests were therefore used to verify that the numerical results were independent of the parameter $h / H^{*}$, the ratio of the layer thickness to the domain size in the $z$ direction, provided sufficiently small values were used $\left(2 \pi H^{*}=15 h\right.$ and $2 \pi L^{*}=30 L$ in most of the cases reported below).

\section{a. Barotropic-baroclinic instability experiments}

In this section we present numerical integrations of BTBC roll-up of a PV layer in order to investigate the behavior as $\Delta q$ is varied, as well as to emphasize the contrast with the KH instability described below. As we are solving the set of Eqs. (20)-(22) in two dimensions, there is an implicit assumption that the evolution of the PV layer will remain $X$ independent as the layer rollsup in the $y-z$ plane. As previously discussed, we believe this is a reasonable assumption as the $x$ direction intersects stratification surfaces, which are likely to act to suppress instability. It should be clear that, in order to rigorously test the validity of this assumption, full threedimensional simulations would be necessary; these are, however, beyond the scope of the present work.

BTBC instability involves balanced flow, in the sense that the evolution may be described by the slow, balanced scaling (4) and we therefore may solve (20)-(22) with $\delta=f / N, \epsilon=1$, and $t \sim f^{-1}$. It is to be emphasised, however, that the balance in question corresponds to that assumed in quasigeostrophic theory only in the limit $\Delta q \rightarrow 0$. In all the experiments, Prandtl's ratio is set to $\operatorname{Pr}=f / N=1 / 100$. The analysis of section 4a suggests 

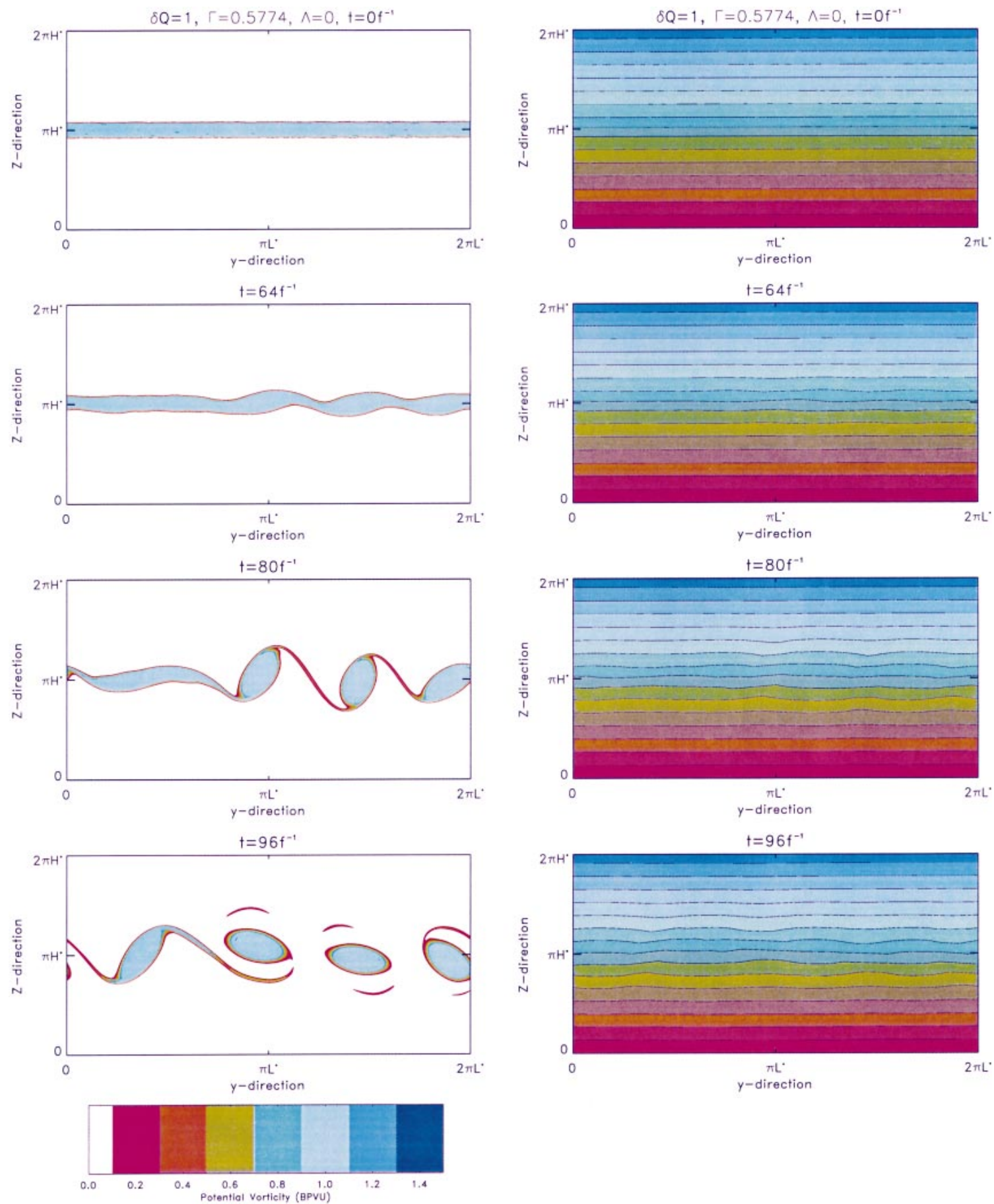

FIG. 3. BTBC instability with $\Delta q=1, \alpha=30^{\circ}$ (corresponding to $\Gamma=0.57735$ ), and $\Lambda=0$. (left) Anomalous PV $\Delta q$ (with nondimensional values indicated by the color bar). (right) Total buoyancy $\bar{b}+b$, with contour intervals $0.4 N^{2} H$; recall that the absolute value of buoyancy is arbitrary, hence the coloring scheme is used for illustration only.

that tilting of the layer will not have an important qualitative influence on the dynamics of BTBC instability, so for the experiments in this section we take $\Lambda=0$ and $\alpha=30^{\circ}(\Gamma \approx 0.57735)$. A series of integrations with $\Delta q=0.5,1,2$, and 4 is performed.

Figure 3 shows the results from the experiment with
$\Delta q=1$. Waves eventually develop on the PV layer (by $t=64 f^{-1}$ ), and the layer is observed to roll-up into a series of four "PV tubes" over the next 20-30 inertial times (dimensionally, one inertial time $f^{-1}$ at $45^{\circ} \mathrm{N}$ is approximately $2.7 \mathrm{~h}$ ). Potential vorticity is materially conserved throughout the experiment to a good ap- 

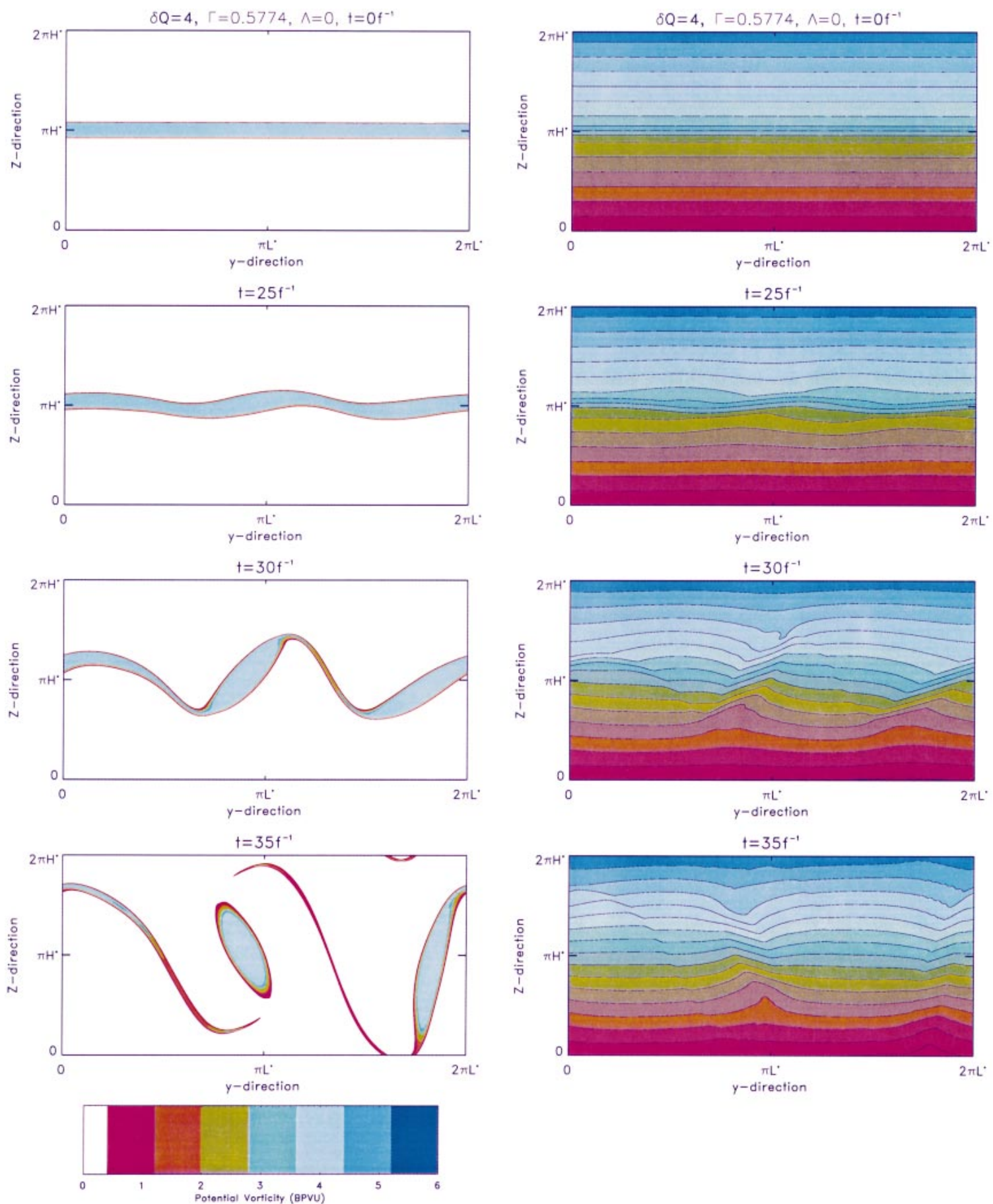

FIG. 4. As in Fig. 3, but for $\Delta q=4$.

proximation, except in the regions between the $\mathrm{PV}$ tubes where relatively high strain causes the layer to be thinned to the grid scale. It is notable that despite the overturning of PV contours during the roll up the total buoyancy field (Fig. 3, right) remains in balance; that is, buoyancy surfaces are only weakly perturbed from their initial state, with their configuration determined entirely by the PV distribution. Furthermore, snapshots of the buoyancy field reveal that far from the PV layer the buoyancy contours remain almost unperturbed throughout the roll up, suggesting that the roll up is accompanied by very little radiation of gravity waves.

Figure 4 shows evolution of the instability for $\Delta q=$ 4. As expected the instability develops faster than for 


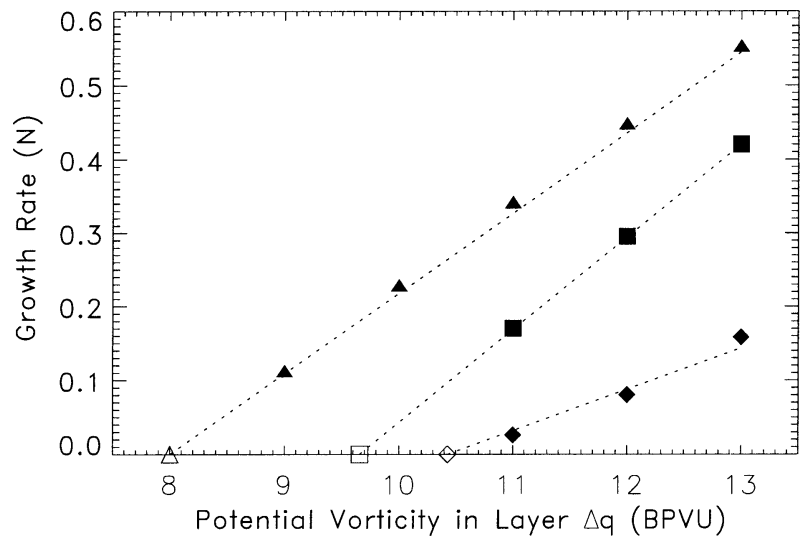

FIG. 5. The growth rate of the most unstable mode vs the anomalous PV $\Delta q$, for three different angles of orientation: $\alpha=15^{\circ}$ (diamonds), $30^{\circ}$ (triangles), and $45^{\circ}$ (squares). The unfilled points on the $x$ axis indicate the marginal stability value for each of the three cases, as determined by the analysis of section 4 ; these values are $\Delta q=8$ (for $\left.\alpha=30^{\circ}\right) ; 4(1+\sqrt{2})$ (for $\left.\alpha=45^{\circ}\right)$; and $8(\kappa+2 \sqrt{\kappa})$, where $\kappa=$ $2-\sqrt{3}$ (for $\alpha=15^{\circ}$ ).

$\Delta q=1$ (the quasigeostrophic theory discussed above suggests that the time scale for growth is proportional to $\left.\Delta q^{-1}\right)$. However, $\Delta q=4$ is far from the quasigeostrophic limit $(\Delta q \rightarrow 0)$, and one consequence of this is that the fastest growing normal mode for the PV layer has doubled in wavelength compared with the $\Delta q=1$ case. As a result, the PV tubes that form appear more elliptical. Another contrast with Fig. 3 is the degree to which the surfaces of constant buoyancy are tilted during the roll up of the PV layer. Although the slopes of the buoyancy surfaces appear large compared to those in Fig. 3, they nevertheless remain in balance with the $\mathrm{PV}$ distribution, and the physical slope remains $O(\operatorname{Pr})$ at all times.

\section{b. Kelvin-Helmholtz instability experiments}

In this section we describe experiments that are either initialized in the $\mathrm{KH}$ unstable region of parameter space shown in Fig. 2 or are tilted into the unstable region by the action of vertical shear. For this reason we use the fast scalings for length and time scales (5) so that $\delta=$ $1, \epsilon=f / N$, and $t \sim N^{-1}$. As above, we set $\operatorname{Pr}=1 /$ 100. Note that $\mathrm{KH}$ instability also occurs if the slow scaling is chosen, but that numerical problems result because the $\mathrm{KH}$ billows are not resolved either in space or time. BTBC instability, however, will not develop on the short horizontal length scale of the fast scaling and, in any case, would not be important on the short time scale of the following experiments.

In Fig. 5, numerically calculated growth rates of the fastest growing unstable $\mathrm{KH}$ mode are illustrated for a range of values of $\Delta q$ (from 9 to 13) and for three different constant angles of tilt ( $\alpha=15^{\circ}, 30^{\circ}$, and $45^{\circ}$, and $\Lambda=0$ ). The growth rates are obtained directly from model experiments (indicated by the symbols in Fig. 2) by isolating the period where $\mathrm{KH}$ waves are growing exponentially and identifying the wavenumber with the fastest growth rate. On the abscissa we have also plotted the point of marginal stability calculated analytically for each angle of tilt, following the arguments outlined in section 4 . The numerical growth rate results are consistent with the locations of the predicted stability boundaries $\Delta q_{c}(\alpha)$, with the growth rate $\omega_{i}$ of the fastest growing $\mathrm{KH}$ wave approximately satisfying $\omega_{i} \sim[\Delta q$ $\left.-\Delta q_{c}(\alpha)\right]$. In the numerical experiments, the length scale associated with the fastest growing $\mathrm{KH}$ wave is also found to be strongly dependent on the angle of tilt $\alpha$. The wavelength of the fastest growing wave was found numerically to be 22.53, 10.40, and $6.44 \mathrm{H}=$ $f L_{x} / N$ for $\alpha=15^{\circ}, 30^{\circ}$, and $45^{\circ}$, respectively. This dependency on $\alpha$ largely reflects the fact that the vertical scale of the layer in physical coordinates equals $H \Gamma^{-1}$, and hence decreases as the layer is tilted by the shear flow.

The most interesting case, where an initially stable PV layer is tilted by the ambient shear into a KH unstable regime, is illustrated in Fig. 6. The initial parameter settings are $\Delta q=12$ and $\Gamma_{0}=0.2$ (corresponding to $\alpha \approx 11.3^{\circ}$ ); the constant shear $\Lambda=0.1$. The change in $\alpha$ during the experiment is indicated by the arrow in Fig. 2. At the beginning of the experiment, the layer is stable, and the initial noise added to perturb the layer does not lead to exponential growth. As the layer is tilted, however, it enters the unstable regime and exponential growth commences. Eventually, fully nonlinear KH billows develop, as seen in the $t=125 \mathrm{~N}^{-1}$ and $137.5 N^{-1}$ panels. These act to mix mass and momentum across the region of the PV layer (e.g., Scinocca 1995). The final state in the $t=150 N^{-1}$ panel shows the layer to be fully turbulent and thoroughly mixed.

The most striking qualitative difference between the development of a $\mathrm{KH}$ instability and the balanced BTBC instability described above, leaving aside the different length and time scales on which they develop, is clear from Fig. 6: KH instability leads to overturning buoyancy surfaces, whereas BTBC instability does not. Sharp gradients develop in the buoyancy field within the overturning $\mathrm{KH}$ billows, and dissipation causes PV to no longer be materially conserved within the layer. In our model this dissipation is numerical, but PV nonconservation within actual $\mathrm{KH}$ billows would also occur since, no matter how low the thermal diffusivity, buoyancy gradients inside the billows will eventually become steep enough for diffusion to become important. At the end of the numerical experiment $\left(t=150 N^{-1}\right)$, the PV field is rather noisy, and the mean PV in the final layer has a mean value of $\Delta q \approx 6$. Note that this final mean value of PV does not depend on the specific details of the diffusion (numerical or physical) because the total PV substance integrated between two unbroken buoyancy surfaces, above and below the layer, must remain constant throughout the evolution of the instability (Haynes and McIntyre 1987). Thus, the final mean PV 

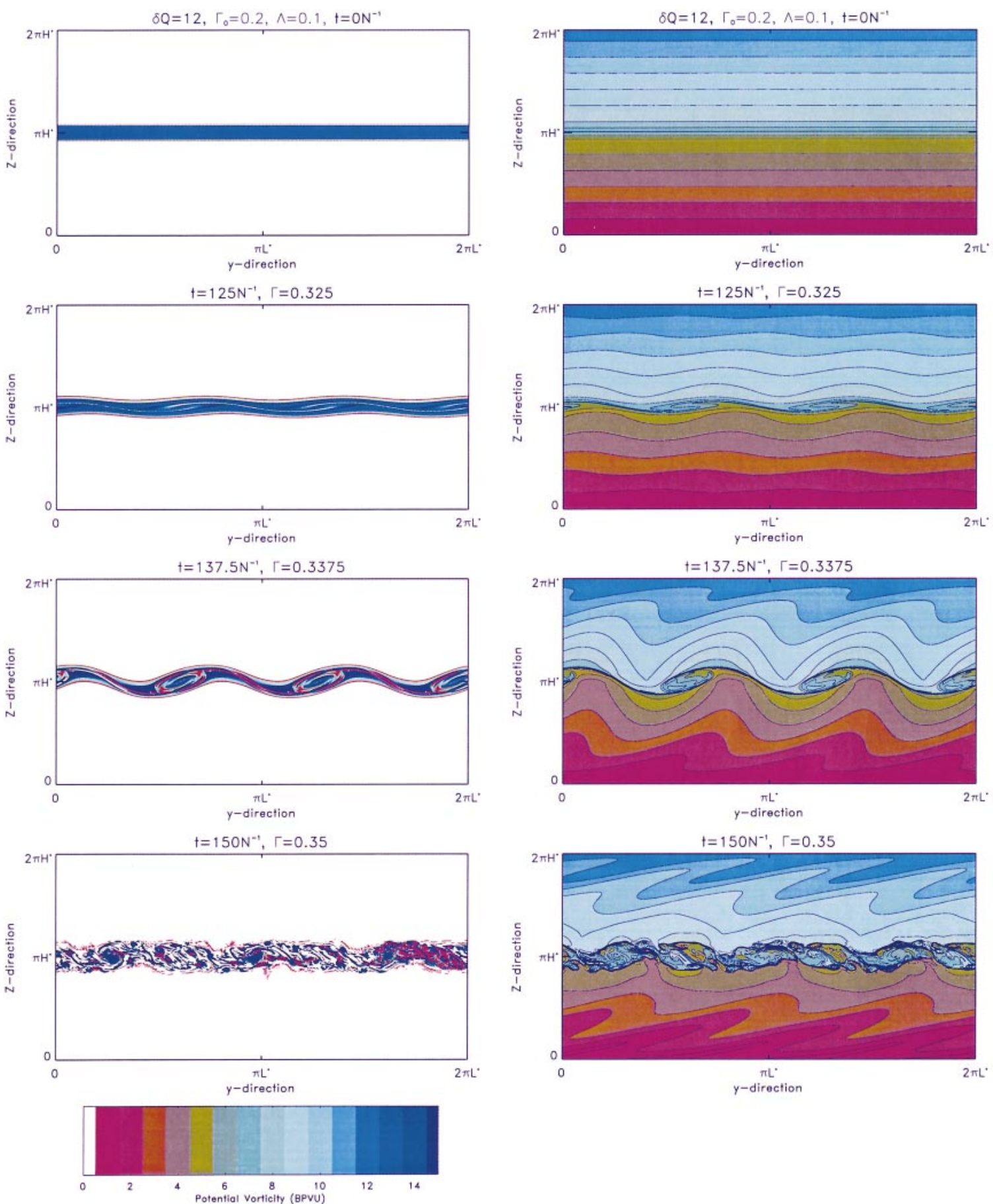

FIG. 6. Kelvin-Helmholtz instability with $\Delta q=12$. The ambient shear is $\Lambda=0.1$, and the initial angle of tilt is $\alpha_{0} \approx 11.3^{\circ}$ (corresponding to $\Gamma_{0}=0.2$ ). As in Fig. 3, the left panels show $\Delta q$ (with values indicated by the color bar), and the right panels show the total buoyancy $\bar{b}+b$ with contour interval $0.4 N^{2} H$.

value in the layer must be determined by the amount of entrainment occurring during the $\mathrm{KH}$ roll up.

The evolution of the Richardson number and the exponential growth rate of the fastest growing mode during the $\mathrm{KH}$ instability are shown in Fig. 7. The growth rate is zero until the Richardson reaches the value of
0.22 , after which point the growth rate starts to increase as the layer tilts and Ri decreases further, until nonlinear saturation of growth occurs late in the experiment. The reason why exponential growth does not begin until $\mathrm{Ri}$ $=0.22$, as opposed to the canonical value $\mathrm{Ri}=0.25$, is that the scale of the periodic domain causes a dis- 


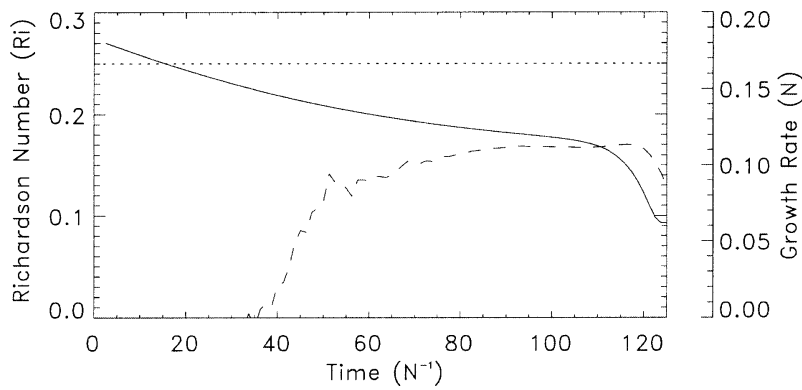

FIG. 7. Time evolution of the Richardson number (solid curve) and the exponential growth rate of the wave-3 disturbance to the layer (dashed curve), for the experiment in Fig. 6. The dotted line indicates the Miles-Howard stability boundary at Ri $=1 / 4$.

cretization of the horizontal wavenumbers in our experiment. For the linear growth rate calculations reported for Fig. 5, the domain was extended to minimize this effect. However, for the experiment in Figs. 6 and 7 , the priority was to resolve the $\mathrm{KH}$ billows.

\section{Discussion and conclusions}

Having considered in detail the dynamics of tilted layers of anomalous PV, we now discuss the implication of our results for the atmosphere. The key to this lies in determining where in the parameter diagram of Fig. 2 typical atmospheric PV layers are likely to be located. In particular, one would like to determine whether observed PV layers are likely to exceed the threshold $\Delta q$ $=8$ required for $\mathrm{KH}$ instability. This appears unlikely for layers in the winter stratospheric surf zone, as the contrast in PV between the polar vortex and the surf zone is such that $\Delta q$ will seldom exceed 1.5-2 BPVU (Waugh et al. 1994). For this reason, we have concentrated on tropospheric PV layers in the following, as a wider range of $\Delta q$ values are likely to be encountered there.

Stratosphere-troposphere exchange events that may lead to the formation of PV layers occur on a wide range of spatial scales, in both the Tropics and the extratropics. Some of the largest-scale events $(H \sim 5 \mathrm{~km}, L \sim 1000$ $\mathrm{km}$ ) are observed in the Tropics in the vicinity of the so-called "westerly duct" (Waugh and Polvani 2000; Waugh and Funatsu 2003); background PV is low in the Tropics, and thus large values of $\Delta q$ are more likely in such events. Extratropical events, such as the Cho et al. (1999) tropopause fold discussed below, may approach similar spatial scales, with the important distinction that extratropical layers may be advected isentropically toward the ground, whereas in the Tropics PV layers of stratospheric origin will remain aloft. Smaller-scale events $(H \sim 500 \mathrm{~m}, L \sim 50 \mathrm{~km})$ have also been widely studied in the extratropics, such as the relatively thin layers of stratospheric origin observed near the extratropical tropopause, and referred to as "streamers" (e.g., Vaughan et al. 2001). Also, stratospheric values of PV may be present in dry intrusion air masses of frontal circulations (e.g., Browning 1990). In general, however, smaller-scale PV layers will be more strongly affected by turbulent mixing and diabatic heating, thus reducing $\Delta q$ (Haynes and Ward 1993; Forster and Wirth 2000).

We will focus our discussion on a large-scale extratropical tropopause fold observed on 13 October 1997 during the Subsonic Assessment (SASS) Ozone and Nitrogen Oxides Experiment (SONEX) aircraft campaign. This example is chosen because Cho et al. (1999) discuss in detail aircraft observations of $\mathrm{KH}$ waves in this fold. It is therefore of interest to assess whether the orientation and magnitude of the anomalous PV is within the unstable $\mathrm{KH}$ range derived in section $4 \mathrm{~b}$ above.

The tropopause fold is illustrated in Fig. 8. The quantity contoured is reverse-domain-filling (RDF) European Centre for Medium-Range Weather Forecasts (ECMWF) operational analysis PV, that is ECMWF PV evaluated 2 days previously along back trajectories started from a regular grid (e.g., Sutton et al. 1994). RDF $\mathrm{PV}$ is used in preference to instantaneous PV as the latter is diffused artificially during the model assimilation on scales greater than that of the fold. Figure 8 shows the a horizontal map of the RDF PV on the 310$\mathrm{K}$ isentropic surface. The fold appears as a long filament of high PV air that has been advected over North America (Hudson Bay can been seen in the map superimposed on Fig. 8). We will concentrate on the SW-NE part of the fold lying in the region of the two sections (X1) and (X2).

Longitude-height sections of Ertel PV along (X1) and (X2) are shown in Fig. 9, and illustrate the horizontal and vertical extent of the PV layer that constitutes the fold. The first of these sections (X1) is at the latitude of the flight $\left(39^{\circ} \mathrm{N}\right)$, and the location of the measured $\mathrm{KH}$ waves is marked with a black square. There is a good correspondence between our RDF PV in the (X1) section and measurements from the ozone lidar on board the aircraft (cf. Plate 3 of Cho et al. 1999). The section (X2) at $49^{\circ} \mathrm{N}$ illustrates the latitudinal extent and coherence of the PV layer.

Figure 10 shows plots of RDF PV against longitude, at a height of $7 \mathrm{~km}$, and at the respective latitudes of the sections (X1) and (X2). These reveal that the RDF PV reaches values of 7-9 potential vorticity units (PVUs; $1 \mathrm{PVU}=1 \times 10^{-6} \mathrm{~m}^{2} \mathrm{~s}^{-1} \mathrm{~K} \mathrm{~kg}^{-1}$ ) in the layer, whereas background values are found to be in the range $0.3-1.0$ PVU. Thus, $\Delta q$ would be in the range 7-30 in background PV units. In addition, the aspect ratio of the PV layer is around 80:1 suggesting a value for $\alpha$ close to $30^{\circ}$ (taking 100:1 as a typical tropospheric value of $N: f$ ). We therefore conclude that $\mathrm{KH}$ instability due to the tilting of the PV layer is a plausible explanation for the observed $\mathrm{KH}$ waves. This raises the possibility that such $\mathrm{KH}$ instability may occur systematically in many stratosphere-troposphere exchange events, and may thus provide a mechanism for rapid mixing of stratospheric chemical species into the troposphere. 

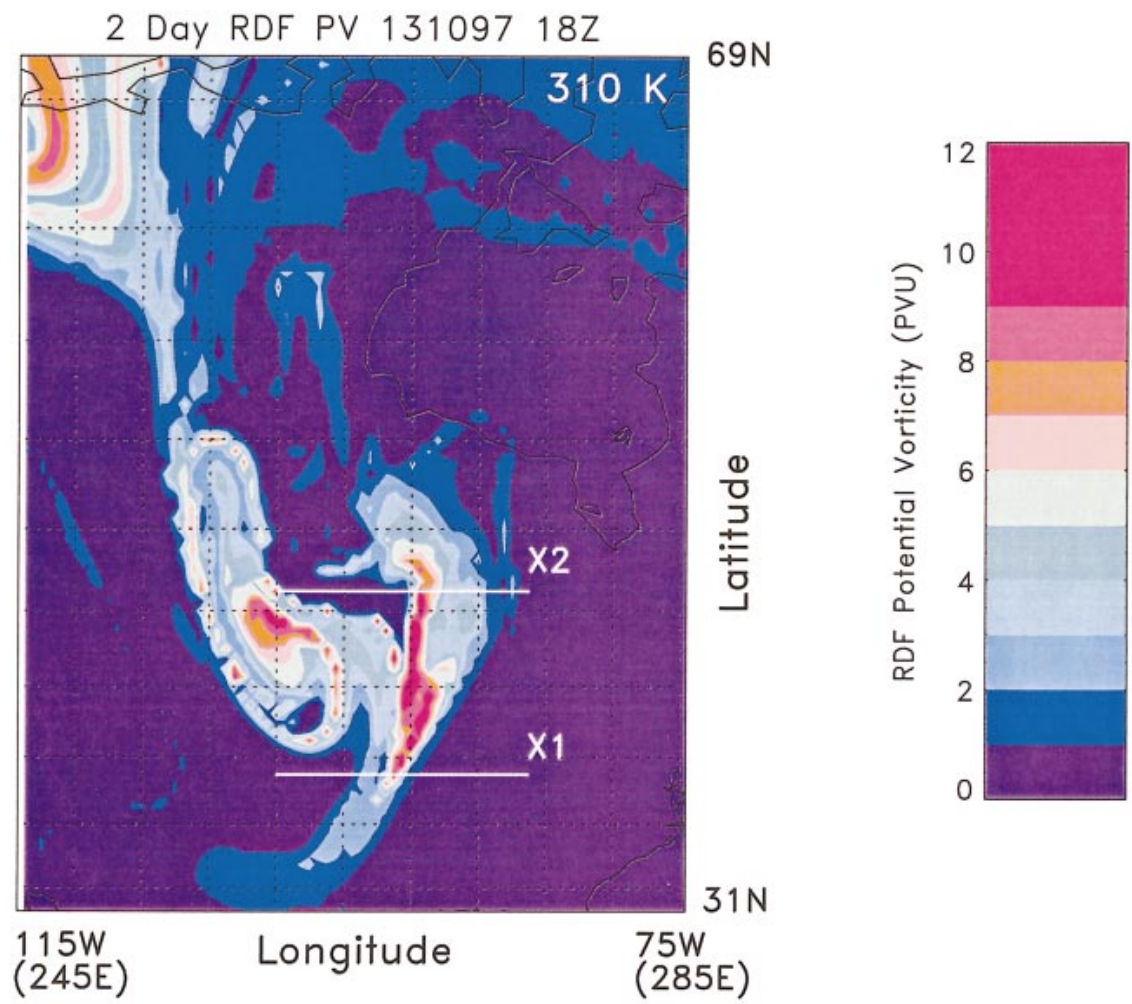

FIG. 8. Ertel PV on the 310-K isentropic surface at 1800 UTC 13 Oct 1997, for the tropopause fold discussed in Cho et al. (1999). The PV is here calculated using the RDF technique, at 2 days previously along back trajectories started on a $0.5^{\circ} \times 0.5^{\circ}$ grid. The color code is based on PVUs.
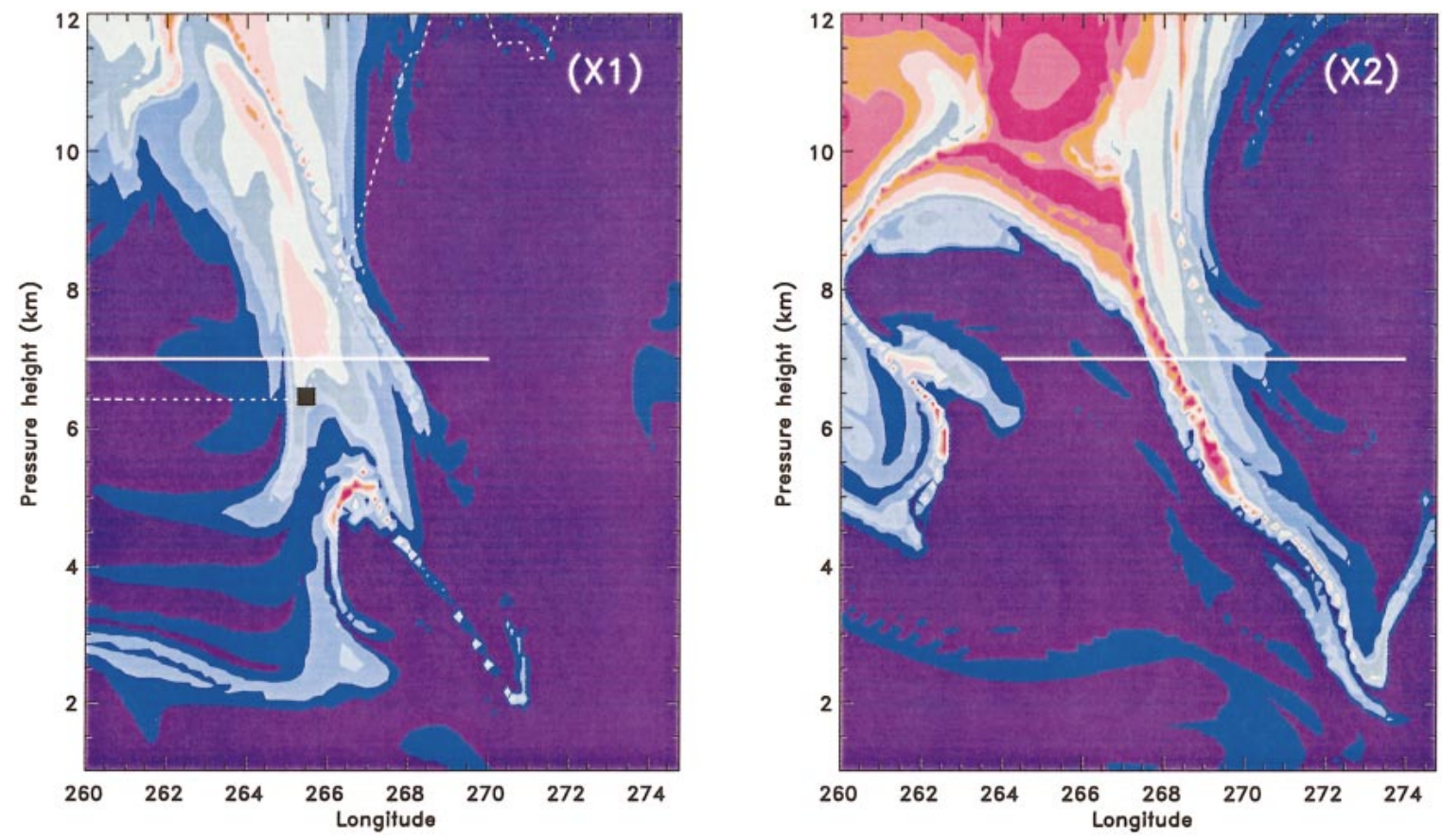

FIG. 9. Ertel PV in the tropopause fold, along two longitude-height sections, as indicated by the solid white lines in Fig. 8. (left) Cross section along $(\mathrm{X} 1)$ at latitude $39^{\circ} \mathrm{N}$. The dotted line shows the trajectory of the SONEX flight, and the black square the location of the measured $\mathrm{KH}$ waves. (right) Cross section along $\left(\mathrm{X} 2 ; 49^{\circ} \mathrm{N}\right)$. As in Fig. 8, the PV is computed using the RDF technique. 

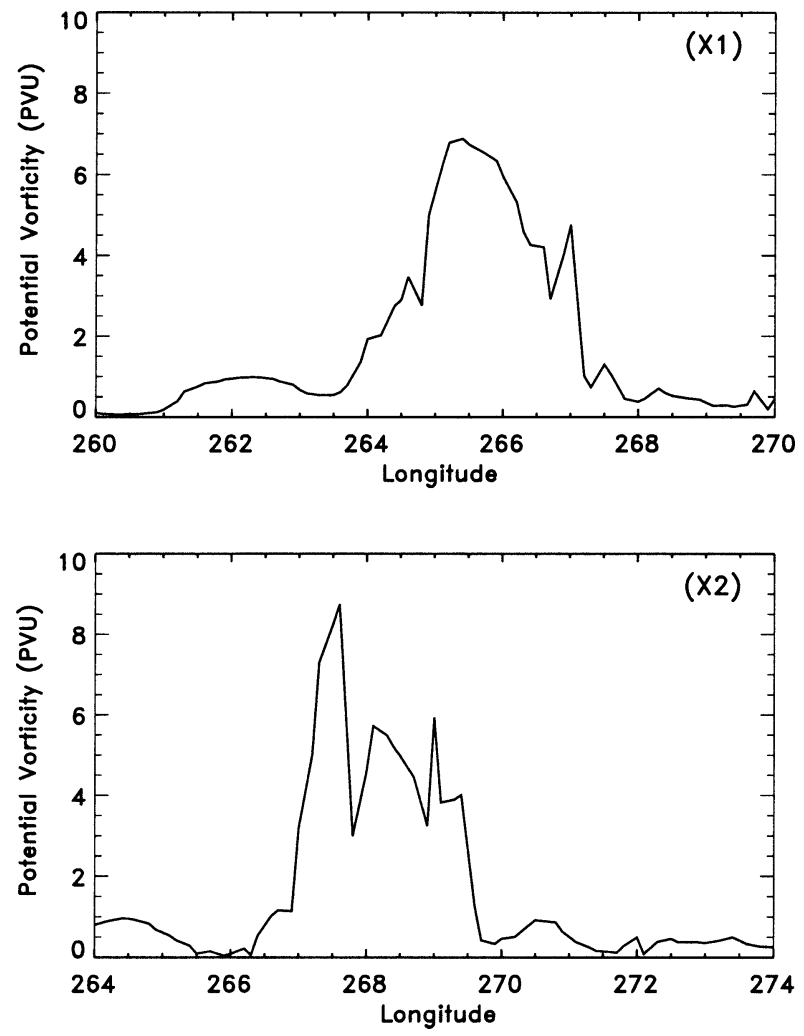

FIG. 10. Ertel PV vs longitude, at constant latitude and height, along the two horizontal sections indicated by the solid lines in Fig. 9. The two sections are at $\left(\mathrm{X} 1 ; 39^{\circ} \mathrm{N}\right)$ and $\left(\mathrm{X} 2 ; 49^{\circ} \mathrm{N}\right)$, and are both at height $7 \mathrm{~km}$.

Beyond the KH instability, we note that another potential application of this work arises in the context of PV tubes. We have shown that they can arise from tilted PV layers as a result of BTBC instability, as is illustrated in Figs. 3 and 4. Considerable evidence for the existence of these tubes, at least in general circulation models, was presented in Bithell et al. (1999), who showed that such tubes arise in connection with tropopause folds (see especially their Fig. 6). Note that the formation of such tubes is a fundamentally different process from the roll up of tropopause anomalies described by Wirth et al. (1997). In the latter the tropopause is represented by infinite jump in PV, but is perturbed only infinitesimally in the vertical direction. That model (see also Juckes 1995 ) is, by construction, concerned with the dynamics of a weakly distorted tropopause, whereas the dynamics of PV layers we have considered in this paper are relevant to the situation where the tropopause has been strongly distorted, for example, by Rossby wave breaking.

Finally, we note that the sudden onset of $\mathrm{KH}$ instability to the tilting balanced state described in section 3 offers a clean example of a sudden departure from the balanced slow manifold leading to fast, unbalanced motion. The stripping of layers of high PV from the tropopause, followed by tilting due to vertical shear, therefore provides a continuous, ongoing mechanism for the spontaneous in situ generation of unbalanced motion, and hence clear air turbulence, in the tropopause region.

Acknowledgments. JGE was supported by NERC Fellowship NER/I/S/1999/00137. LMP is supported, in part, by the U.S. National Science Foundation.

\section{REFERENCES}

Appenzeller, C., H. C. Davies, and W. A. Norton, 1996: Fragmentation of stratospheric intrusions. J. Geophys. Res., 101, 1435-1456. Bartello, P., 1995: Geostrophic adjustment and inverse cascades in rotating stratified turbulence. J. Atmos. Sci., 52, 4410-4428.

Bithell, M., L. J. Gray, and B. D. Cox, 1999: A three-dimensional view of the evolution of midlatitude stratospheric intrusions. $J$. Atmos. Sci., 56, 673-687.

Browning, K. A., 1990: Organization of clouds and precipitation in extratropical cyclones. Extratropical Cyclones-The Erik Palmén Memorial Volume, C. Newton and E. O. Holopainen, Eds., Amer. Meteor. Soc., 129-153.

Cho, J. Y. N., and Coauthors, 1999: Observations of convective and dynamical instabilities in tropopause folds and their contribution to stratosphere-troposphere exchange. J. Geophys. Res., 104, 21 54921568.

Drazin, P. G., and W. H. Reid, 1981: Hydrodynamic Instability. Cambridge University Press, 527 pp.

Dritschel, D. G., 1989: On the stabilization of a two-dimensional vortex strip by adverse shear. J. Fluid Mech., 206, 193-221.

— , P. H. Haynes, M. N. Juckes, and T. G. Shepherd, 1991: The stability of a two-dimensional vorticity filament under uniform strain. J. Fluid Mech., 230, 647-665.

Esler, J. G., L. M. Polvani, and R. A. Plumb, 1999: On the mix-down times of dynamically active potential vorticity filaments. Geophys. Res. Lett., 26, 2953-2956.

Ford, R., M. E. McIntyre, and W. A. Norton, 2000: Balance and the slow quasimanifold: Some explicit results. J. Atmos. Sci., 57, $1236-1254$.

Forster, C., and V. Wirth, 2000: Radiative decay of idealized stratospheric filaments in the troposphere. J. Geophys. Res., 105, $10169-10184$

Hakim, G. J., C. Snyder, and D. J. Muraki, 2002: A new surface model for cyclone-anticyclone asymmetry. J. Atmos. Sci., 59, $2405-2420$.

Haynes, P. H., and M. E. McIntyre, 1987: On the evolution of vorticity and potential vorticity in the presence of diabatic heating and frictional or other forces. J. Atmos. Sci., 44, 828-841.

_ on potential vorticity structures, including the influence of background shear and strain. J. Atmos. Sci., 50, 3431-3453.

Hoskins, B. J., M. E. McIntyre, and A. W. Robertson, 1985: On the use and significance of isentropic potential vorticity maps. Quart. J. Roy. Meteor. Soc., 111, 877-946.

Howard, L. N., 1961: Note on a paper of John Miles. J. Fluid Mech., 10, $509-512$.

Juckes, M. N., 1995: Instability of surface and upper tropospheric shear lines. J. Atmos. Sci., 52, 3247-3262.

McIntyre, M. E., and T. N. Palmer, 1984: The "surf zone" in the stratosphere. J. Atmos. Terr. Phys., 46, 825-849.

— hemisphere. J. Atmos. Sci., 57, 1214-1235.

Miles, J. W., 1961: On the stability of heterogeneous shear flows. $J$. Fluid Mech., 10, 495-508.

Polvani, L. M., and R. Saravanan, 2000: The three-dimensional structure of breaking Rossby waves in the polar wintertime stratosphere. J. Atmos. Sci., 57, 3663-3685.

Rayleigh, J. W. S., 1894: The Theory of Sound. 2d ed. Macmillan, $186 \mathrm{pp}$. 
Scinocca, J. F., 1995: The mixing of mass and momentum by KelvinHelmholtz billows. J. Atmos. Sci., 52, 2509-2530.

Sutton, R., H. Maclean, R. Swinbank, A. O'Neill, and F. W. Taylor, 1994: High resolution tracer fields estimated from satellite observations using Lagrangian trajectory calculations. J. Atmos. Sci., 51, 2995-3005.

Vaughan, G., H. Gouget, F. M. O'Connor, and D. Wier, 2001: Fine scale layering on the edge of a stratospheric intrusion. Atmos. Environ., 35, 2215-2221.

Waugh, D. W., and D. G. Dritschel, 1991: The stability of filamentary vorticity in two-dimensional geophysical vortex-dynamics models. J. Fluid Mech., 231, 575-598.
- , and L. M. Polvani, 2000: Intrusions into the tropical upper troposphere. Geophys. Res. Lett., 27, 3857-3860.

_- and B. M. Funastu, 2003: Intrusions into the tropical upper troposphere: Three-dimensional structure and accompanying ozone and OLR distributions. J. Atmos. Sci., 60, 637-653.

__ , and Coauthors, 1994: Transport of material out of the stratospheric Arctic vortex by Rossby wave breaking. J. Geophys. Res., 99, 1071-1088.

Wirth, V., Ch. Appenzeller, and M. N. Juckes, 1997: Signatures of induced vertical air motion accompanying quasi-horizontal rollup of stratospheric intrusions. Mon. Wea. Rev., 125, 2504-2519. 